\title{
Análisis de los factores que afectan a la decisión de ser emprendedor
}

\author{
M. Jesús Freire Seoane \\ Directora do Observatorio Ocupacional \\ Universidade da Coruña \\ 15011 A Coruña \\ maje@udc.es
}

\author{
Mercedes Teijeiro Álvarez \\ Departamento de A.D.E \\ Universidade da Coruña \\ mteijeiro@udc.es
}

\begin{abstract}
RESUMEN
Una parte importante de la salud de una economía se refleja en la evolución de su mercado de trabajo. Conocer las características de sus principales variables es fundamental para realizar un diagnóstico de la inserción laboral. Esta investigación trata de analizar las diferencias entre las personas que trabajan por cuenta ajena y los emprendedores. El objetivo de este trabajo es analizar si existen factores que impulsen la decisión individual de ser emprendedor. El estudio se lleva a cabo con microdatos procedentes de una encuesta realizada en el año 2008 en Galicia, entre jóvenes con edades comprendidas entre 16 y 34 años. En el análisis se profundiza en una serie de rasgos básicos de los individuos de la muestra y los más significativos son: el nivel de estudios, el método de búsqueda de empleo, la intensidad en la búsqueda, la precariedad en el trabajo y el tipo de relación laboral. Se han obtenido conclusiones significativas con un modelo logit segregando entre estos dos grupos de trabajadores.
\end{abstract}

Palabras clave: inserción laboral, trabajadores por cuenta ajena, emprendedores.

Clasificación JEL: J24, L53, M13. 


\begin{abstract}
The evolution of the Labour market reflects to a great extent the health of an economy. Knowing the characteristics of its main variables is key for the study of labour insertion. In this piece of research we try to analyze the differences between those working for an employer, and those self employed, «the entrepreneurs». The objective of the analysis is to determine whether the decision to become an entrepreneur is influenced by external factors. The analysis is based on the data from a survey carried out in 2008 among population age 16 to 34 year old. The analysis looks into a number of basic characteristic of the sample population, where the most significant are: level of studies, type and intensity of job search, current job circumstances and professional relationships. For the analysis of the differences between both groups we used a segregated logit model. The conclusions that can be drawn from the analysis are significant.
\end{abstract}

Keywords: labour insertion, employee, self-employee

JEL Classification: J24, L53, M13.

\title{
1. INTRODUCCIÓN
}

En la sociedad moderna las primeras referencias sobre el concepto de emprendedor se encuentran en la obra de J. Schumpeter «The theory of economic development: an inquiry into profits, capital credit, interest, and business cycle», donde el autor pone de manifiesto que el emprendedor es un individuo generador de crecimiento económico. A partir de ese momento parece desencadenarse un interés espectacular por el tema como lo demuestran las numerosas investigaciones realizadas sobre el impacto de la actividad emprendedora en las economías de mercado y su influencia en la creación de empleo, el crecimiento económico y, como contrapartida, en el bienestar de la sociedad (Johnson y Loveman, 1995).

El objetivo de este trabajo es analizar el capital humano de los emprendedores gallegos y los factores que les han impulsado a adoptar esta decisión. Ya que tanto la Comisión Europea como las autoridades españolas están demandando un incremento de la actividad emprendedora (Comisión de las Comunidades Europeas, 2003; Acs, Arenius, Hay y Minnití, 2005), al haber introducido en sus directivas de actuación la importancia que este colectivo tiene para el progreso de la economía. En nuestra investigación hemos considerado una visión general del emprendedor definiéndolo como la persona que crea su propia empresa, aportando su trabajo y que asume los riesgos financieros de la misma. 
Uno de los aspectos más preocupantes de la situación laboral en los últimos años es la mayor dificultad a la que se enfrentan los jóvenes para encontrar empleo, este hecho es de una gran repercusión social y económica. La cuestión que hoy preocupa más a la sociedad es el no utilizar de una manera eficiente el capital humano de la población más joven, ya que esta situación puede provocar importantes pérdidas de competitividad en relación al resto de países o regiones y, por consiguiente, afectar a su crecimiento económico. Teniendo en cuenta, sobre todo, la importancia que este colectivo tiene para el progreso de la economía, nuestra investigación se centra en el cálculo del capital humano de los jóvenes gallegos y los factores que les han impulsado a adoptar la decisión de ser emprendedores.

El presente trabajo trata de responder a las siguientes preguntas:

- Qué percepción tienen los jóvenes del mercado laboral y cómo puede afectarles el hecho de ser emprendedor.

- En los procesos de incorporación de los jóvenes al mundo laboral, qué impacto tiene la decisión de crear una empresa

El intento de resolver estas cuestiones nos lleva a analizar si existen factores que afectan a la decisión individual de ser emprendedor o trabajador por cuenta ajena. Desde esta última perspectiva, el actual trabajo pretende ampliar el estudio del perfil del emprendedor aplicándolo a los jóvenes de 16 a 34 años (ambos incluidos) de diferentes comarcas de la Comunidad Autónoma Gallega, basándonos en las variables personales, sociales, y en los procesos de incorporación al mercado laboral.

En el contexto actual el perfil del emprendedor europeo medio es: ser varón, de aproximadamente 35 años, que previamente haya trabajado en una empresa pequeña, en un puesto de trabajo de dirección (mando intermedio) o como trabajador cualificado y con nivel de formación y experiencia medio. Las motivaciones que le han impulsado a crear su propia empresa han sido principalmente, la autorrealización, el deseo de independencia y el autoempleo.

El trabajo se organiza del siguiente modo: en primer lugar, se define la base de datos que se ha utilizado, ya que supone una novedad importante en relación con la mayoría de los trabajos sobre inserción laboral. En segundo lugar, se realiza una presentación sumaria de la inserción laboral de los jóvenes encuestados, lo que permitirá destacar algunos hechos y tendencias aparentemente elementales, pero que es necesario tener en cuenta como punto de partida. Se trata de una relación significativa con el sexo, la edad, el nivel educativo y el estado civil. En el apartado 3 se abordan las características de la inserción laboral de los jóvenes gallegos. En el apartado 4 se analizan los aspectos 
más valorados del trabajo y el nivel de satisfacción con el mismo. En el apartado 5 se presentan las estimaciones del modelo logit utilizado y se interpretan los resultados. Se trata, sin duda, de la parte más técnica de esta investigación. Por último, el trabajo se cierra con una recopilación de las principales conclusiones.

\section{REVISIÓN DE LA LITERATURA}

En las últimas décadas se ha venido prestando una especial atención a lo que se ha denominado «entrepreneurship», término que se ha traducido, generalmente por «emprendedor». Este concepto se aplica, generalmente a las personas que desean crear y llevar a cabo un negocio, esto es, desarrollar una actividad productiva. No cabe duda de que la creación de empresas constituye un elemento fundamental en la dinámica económica de cualquier región o país y que su evolución depende tanto de factores personales como materiales. Por tanto, es lógico que el espíritu emprendedor sea uno de los nuevos campos de investigación en el área de la Economía.

El término emprendedor no es un concepto nuevo ya que los autores clásicos hacían referencia a él, pero hay que esperar hasta 1936 cuando Schumpeter renueva el concepto y considera que se trata de uno de los motores principales del crecimiento económico. Si bien desde entonces el concepto permanecía latente, fue a partir de los años 80 cuando comenzó el espectacular desarrollo que llega hasta nuestros días.

Los trabajos empíricos realizados en estas últimas décadas por numerosos autores sobre las diferentes corrientes teóricas ponen de manifiesto que existen problemas metodológicos y estadísticos debido a la ausencia de un paradigma que respalde la coherencia de las investigaciones (Gartner, 1988; Cooper y Gascón, 1992; Slevin y Covin, 1995). Otros autores como Aldrich y Baker (1997) están de acuerdo al afirmar que el campo de la creación de empresas ha progresado poco, consideran que tan sólo ha pasado de un estado de disciplina a ser considerado como un marco conceptual de ciencia. Al tratarse de un tema inter-disciplinar, el estudio de la conducta emprendedora se estudia tanto desde las características personales de los individuos como desde el punto de vista de las actividades empresariales y sus efectos económicos y sociales, por lo que nos encontramos con multitud de paradigmas que pueden considerarse ambiguos (Low y MacMillan, 1988). Pese a todo, no cabe duda de que hay un creciente consenso sobre la existencia de diferentes causas detrás de los procesos del espíritu emprendedor (Zahara, 1991; MacMillan y Katz, 1992; Naffzinger, 1995 y Paige, 1999).

Aunque estamos en un estadio inicial, existe evidencia de que las recientes investigaciones tienden a converger en modelos y teorías, por lo que hay bases científicas 
para desarrollar trabajos a partir de los iniciados (Ibrayeva, 1999), lo que nos permitirá ir avanzando poco a poco en el concepto de emprendedor.

A lo largo del tiempo se han podido distinguir dos tipos de teorías: unas en relación a la creación de empresas y al empleo y, otras en relación con las oportunidades (Veciana, 1999; Díaz, 2002 y Toledano, 2003). La primera interpretación proviene de la teoría económica y la segunda de las teorías psicológicas.

El primer enfoque es el de la teoría neoclásica en donde la figura del empresario no era tenida en cuenta y se identificaba como el capitalista propietario de los medios de producción (Marshall, 1890; Clarck, 1924). En este período el proceso emprendedor se explica a través del individuo que decide ser emprendedor, basándose en los atributos personales que posee y no en la información que puede tener del mercado y de sus oportunidades de negocio (Khilstrom y Laffont, 1979; Evans y Jovanovic, 1989).

Posteriormente, con la escuela austríaca, el concepto de emprendedor se separa del individuo con propensión a ser emprendedor y se centra en el mercado como un proceso frente a la visión de situación de equilibrio general que existía en la escuela neoclásica.

En el enfoque psicológico existe una primera línea de investigación que trata de explicar las diferencias individuales entre emprendedores y no emprendedores. El objetivo final de este enfoque es identificar y explicar las características personales de los emprendedores, y, a su vez, poder detectar a aquellas personas con un potencial innato para convertirse en emprendedores de éxito. Desafortunadamente, dicha línea de investigación no ha logrado resultados consistentes, recibiendo numerosas críticas tanto metodológicas como teóricas, que han demostrado que los rasgos de personalidad del individuo no son suficientes para predecir la conducta emprendedora (Shaver y Scott, 1991; Ami y otros, 1993; Shane y Venkataraman, 2000 y Baron, 2002).

Esto ha llevado a que surja una segunda línea de investigación cuyo objetivo es el estudio de la intención emprendedora, teniendo en cuenta no sólo variables personales sino también variables sociales, así como la interdependencia existente entre ambas (Alexei y Kolvereid, 1999; Krueger y otros, 2000; Audet, 2002 y Douglas y Shepherd, 2002).

El estudio psicosocial del emprendedor es una línea de investigación de gran interés dada la actual situación socioeconómica y el especial interés que existe en los diferentes entornos para que los individuos aprovechen las oportunidades de negocio existentes en el mercado (Davidsson y Honig, 2002; Gartner, 2002 y García del Junco y otros 2003). En los últimos años se ha generado un gran interés por fomentar el dinamismo y crecimiento de la economía de un área geográfica a través de la promoción de las empresas de nueva creación; dicho interés se debe, principalmente, a que se considera 
una de las fuentes principales de generación de empleo neto (Hohti, 2000; Heshmati, 2001 y Audretsch, 2002), ya que la creación de empleo se concentra cada vez más en las pequeñas y medianas empresas.

Actualmente, el término emprendedor ha evolucionado desde la versión de Schumpeter que enfatizaba en la característica de innovación, considerando la actividad emprendedora como aquélla que supone una innovación en la introducción de un nuevo producto, nueva organización o cambio de procesos.

Por su parte Mises (1949), considera al empresario como aquella persona capaz de descubrir y determinar dónde se está ubicado en el mercado y a dónde se quiere llegar. Dicha visión le permite descubrir oportunidades de mercado con su consiguiente generación de beneficio.

Kirzner (1973) argumenta que la esencia de la definición de empresario ha de ser la capacidad de aprovechar las oportunidades de beneficio, pero que a diferencia de aquél, aprende de los errores cometidos en el pasado, por lo que contribuye a la mejora de la actividad de mercado. Así pues, existe una relación entre las instituciones y los emprendedores que facilita el progreso económico.

El planteamiento de Baumol (1990) diferencia varias clases de emprendedores, unos productivos y otros improductivos. Los primeros sólo buscan los medios para aumentar su riqueza, poder y prestigio, teniendo en cuenta el entorno que les rodea.

Con una perspectiva más actual surge la definición de Wennekers y Thurik (1999), en donde se conceptualiza el espíritu emprendedor como la capacidad manifestada por los individuos, ya sea por ellos mismos o mediante equipos, dentro o fuera de organizaciones existentes, de crear nuevas oportunidades económicas (nuevos productos, nuevas formas de organización, etc), introduciendo sus ideas en los mercados y enfrentándose a la incertidumbre y a otros obstáculos.

En este contexto es relevante señalar el trabajo de Congregado, y otros (2008). En esta investigación se hace referencia a tres aspectos diferentes del concepto general de emprendedor. El primero hace referencia a las personas que desarrollan su propio proyecto empresarial, afectando este proyecto sólo a su persona (autónomos, empresarios sin asalariados o trabajadores independientes).El segundo aspecto del emprendedor es cuando se le considera como una persona que desarrolla y gestiona un proyecto empresarial propio o ajeno, y que crea empleo para terceros (empresarios con asalariados, directivos o gerentes asalariados). Por último, también son emprendedores aquellas personas que desarrollan un proyecto empresarial propio y que da empleo a terceros (empresarios con asalariados). 


\section{DisEÑO DE LA MUESTRA}

Este estudio forma parte de una investigación más genérica realizada por el Observatorio Ocupacional de la Universidad de A Coruña cuyo objetivo es analizar la situación educativa y laboral de los jóvenes gallegos de diversas zonas (Comarcas de Bergantiños y Eume y zonas geográficas de Costa da Morte y Ferrolterra), y su transición desde el sistema educativo al mercado laboral.

Su análisis está centrado en el estudio de los rasgos básicos del joven emprendedor, en particular, si los procesos de incorporación al mercado laboral, las estrategias que utiliza en la búsqueda de empleo, así como la importancia y la satisfacción concedidas al trabajo, pueden resultar significativas a la hora de tomar la decisión de ser emprendedor.

El diseño de la muestra realizada entre el 1 de junio y el 30 de septiembre de 2008 es el siguiente:

1. Ámbito: Comarcas de Bergantiños y Eume, y de las zonas geográficas de Costa da Morte y Ferrolterra.

2. Universo: la población con edades comprendidas entre 16 y 34 años, ambos incluidos.

3. Tamaño muestral: 2.067 jóvenes encuestados, la estratificación se realizó mediante una clasificación cruzada por ayuntamiento y grupos de edad.

4. Muestra objetivo para los jóvenes: El total de la muestra de acuerdo con los objetivos y el diseño fue por ayuntamiento, grupos de edad y género.

5. El error muestral para cada una de las zonas fue del $+/-2 \%$ para un nivel de confianza del $98 \%$.

6. Método: Polietápico con estratificación en la primera fase y afijación no proporcional entre las zonas y proporcional en los ayuntamientos.

Para los totales muestrales se estableció que la muestra no fuese inferior a treinta y cinco unidades en cada ayuntamiento, de modo que se pueden hacer estimaciones para cada uno por separado. También, se ha procurado que se mantenga autoponderada para los distintos grupos de edad, ya que se considera que es importante para el análisis de las variaciones en el comportamiento y en la situación del empleo con distintos niveles de estudios. Siguiendo estos criterios se obtuvieron 2.067 encuestas válidas cuya composición por género y situación laboral se encuentran reflejadas en el cuadro 1. 
CuAdro 1.-Muestra obtenida de los jóvenes respecto a la situación laboral el mes anterior a la entrevista por género

\begin{tabular}{|l|l|r|c|c|}
\hline \multicolumn{2}{|c|}{ Trabaja } & Mujer & Hombre & Total \\
\cline { 2 - 5 } & Cuenta ajena & 485 & 330 & 823 \\
\hline No trabaja & 80 & 109 & 189 \\
\hline \multicolumn{2}{|l|}{ Total } & 561 & 473 & 1.055 \\
\hline
\end{tabular}

En la composición de la muestra por grupos de edad de los encuestados se han considerado cuatro grupos de edad de 16 a 19 años, de 20 a 24 años, de 25 a 29 y de 30 a 34. La distribución de los encuestados por grupos de edad y situación laboral el mes anterior a la entrevista (cuadro 2) nos muestra que el grupo de edad más representativo para el caso de los jóvenes con contrato por cuenta ajena es el de 25 a 29 años con un $36,4 \%$ de la población, mientras que para la submuestra de encuestados con contrato por cuenta propia el grupo de edad más frecuente es el de 30 a 34 años con un 50,3\%. Se observa, como es lógico, que en los grupos de edad más jóvenes la proporción de encuestados que no trabajan o lo hacen sin contrato es mucho más alta, un 40,2\% para el grupo de 16 a 19 años y un 25,2\% para el grupo de 20 a 24 años.

CUADRo 2.-Distribución porcentual de los jóvenes por grupos de edad y situación laboral el mes anterior a la entrevista

\begin{tabular}{|l|c|c|c|c|}
\hline & De 16 a 19 & De 20 a 24 & De 25 a 29 & De 30 a 34 \\
\hline Cuenta ajena & 9,4 & 21,6 & 36,4 & 32,6 \\
\hline Cuenta propia & 1,1 & 16,2 & 32,4 & 50,3 \\
\hline No trabaja & 40,2 & 25,2 & 20,0 & 14,7 \\
\hline
\end{tabular}

En relación a la distribución porcentual de los encuestados por tipo de empleo y estado civil (cuadro 3) se observa que el 32,28\% de los encuestados que trabajan por cuenta propia están casados o en pareja, frente al 28,43\% de los encuestados que trabajan por cuenta ajena.

CUADro 3.-Distribución porcentual de los jóvenes por tipo de empleo y estado civil

\begin{tabular}{|l|c|c|}
\hline & No tener pareja & Tener pareja \\
\hline Cuenta ajena & 71,57 & 28,43 \\
\hline Cuenta propia & 67,72 & 32,28 \\
\hline
\end{tabular}


El cuadro 4 presenta los datos referidos al nivel educativo completado de la muestra de individuos gallegos realizada en 2008 entre la población de 16 a 34 años de edad. El nivel educativo lo clasificamos en 4 tramos:

- Nivel de estudios bajo: que se corresponde con Primaria, Graduado Escolar o sin estudios.

- Nivel de estudios medio: que se corresponde con BUP, ESO, FPI o ciclos de grado medio.

- Nivel de estudios anterior al superior: que se corresponde con COU, Bachillerato, FPII o ciclos de grado superior.

- Nivel de estudios superior: que se corresponde con el título de diplomado o de licenciado.

La interpretación de estos resultados requiere tener en cuenta que en este punto existen dos sesgos de signo contrario, y por tanto, de efecto incierto. En primer lugar, las personas encuestadas, al ser jóvenes, deben tener niveles educativos superiores a los de la población en general. Sin embargo, el hecho de entrevistar a muchos individuos cuyo proceso de formación no ha terminado implica que cuando se les pregunta por el nivel de estudios más elevado que han completado responden con el nivel anterior, con lo cual los resultados finales sobre el nivel de estudios podrían ser inferiores a los de la población total.

Cuadro 4.-Distribución porcentual de los jóvenes por tipo de empleo y nivel de estudios completado

\begin{tabular}{|l|c|c|}
\hline & Cuenta ajena & Cuenta propia \\
\hline Bajo & 22,38 & 26,88 \\
\hline Medio & 19,76 & 24,19 \\
\hline Anterior al superior & 26,31 & 25,81 \\
\hline Superior & 31,55 & 23,12 \\
\hline
\end{tabular}

Estos datos reflejan que el grupo más numeroso en el caso de individuos con contrato por cuenta ajena lo forman los encuestados que han completado el nivel de estudios superior $(31,54 \%)$, mientras que en el caso de los autoempleados el grupo más numeroso lo forman los individuos con nivel de estudios bajo (26,88\%) y nivel de estudios anterior al superior $(25,81 \%)$, mientras que el nivel educativo menos representativo para los emprendedores estudiados es el nivel de estudios superior $(23,12 \%)$. 


\section{EL PROCESO DE INSERCIÓN AL MERCADO LABORAL}

Entendemos la inserción como un proceso complejo, que requiere la consideración de un buen número de variables y la aproximación desde múltiples niveles. Es importante analizar este proceso a nivel macro atendiendo a las condiciones del mercado laboral y a las características de los jóvenes que configuran el capital humano existente. Por otra parte, hay que analizar desde una perspectiva más micro la experiencia de los jóvenes en los diferentes puestos de trabajo que han ido ocupando a lo largo del proceso de inserción y las empresas en las que trabajaban o trabajan. Se requiere también volver la mirada hacia el joven y conocer su preparación y cualificación, sus valores laborales, sus actitudes y sus características personales.

Los procesos de incorporación de los individuos al mundo laboral, y las diversas estrategias que deben desarrollar en la búsqueda de empleo, constituyen una realidad que es preciso analizar desde la importancia que tiene y los efectos que produce. En efecto, la relación jóvenes-empleo suele tratarse desde una óptica objetiva que enfoca el problema, fundamentalmente, desde las estadísticas de empleo y el análisis del comportamiento de los indicadores más relevantes del mercado. Pero, además de esta información facilitada por los indicadores, el resto de los análisis que se podrían llevar a cabo sobre las conductas, las expectativas del mercado y las percepciones que guían la vida personal y colectiva de los jóvenes, así como otras cuestiones de mayor alcance, quedan relegadas o excluidas de la reflexión.

La actividad de búsqueda de empleo consiste en la utilización de diferentes procedimientos con el objetivo de encontrar un puesto de trabajo. Sin embargo, no todos los procedimientos utilizados tienen el mismo grado de eficacia, ni su distribución es homogénea en distintos ámbitos geográficos y fases del ciclo económico. Para analizar estos procedimientos se les ha preguntado a los jóvenes que estaban trabajando en el mes anterior a la encuesta, si han buscado empleo en los últimos cinco años y en caso de que así fuera, cuáles han sido los tres procedimientos de búsqueda de empleo más utilizados, el cuadro 5 nos muestra los resultados por tipo de empleo.

CuADro 5.-Distribución porcentual de los tres métodos más utilizados en la búsqueda de empleo por tipo de empleo

\begin{tabular}{|l|c|c|c|c|c|c|}
\hline & INEM & Currícula & Oposiciones & Contactos & Autoempleo & Otros \\
\hline Cuenta ajena & 45,4 & 59,0 & 13,0 & 57,2 & 2,1 & 17,4 \\
\hline Cuenta propia & 38,4 & 32,6 & 0,8 & 57,6 & 52,9 & 12,2 \\
\hline
\end{tabular}


Destacan especialmente varios hechos, el alto porcentaje del uso de las cartas o currícula a empresas como procedimiento de búsqueda que toma valores muy dispares entre los trabajadores por cuenta ajena (59\%) y los trabajadores por cuenta propia $(32,6 \%)$, así como el prácticamente inexistente porcentaje $(0,8 \%)$ de jóvenes que trabajan por cuenta propia y que su método de búsqueda de empleo ha sido preparar oposiciones. Por otro lado, y aunque evidente, se observa el importante peso del autoempleo $(52,9 \%)$ como método de búsqueda de empleo entre los trabajadores por cuenta propia. El procedimiento de búsqueda de empleo más utilizado tanto para los encuestados que trabajan por cuenta ajena como por cuenta propia ha sido el de promover los contactos con porcentajes que van del $57,2 \%$ al $57,6 \%$ respectivamente.

El cuadro 6 muestra los factores que los jóvenes encuestados citan como los que más influyen a la hora de encontrar empleo. En este caso se les planteó la posibilidad de que marcasen hasta tres factores.

Los datos muestran que un $63,7 \%$ de los jóvenes encuestados con contrato por cuenta propia marcan los contactos de sus padres o amigos como uno de los factores más influyente para encontrar un empleo, frente al $57,1 \%$ de los encuestados con contrato por cuenta ajena. Destacan el alto porcentaje de encuestados con contrato por cuenta propia, un $27,9 \%$, que consideran que el hecho de asumir riesgos es un factor importante a la hora de encontrar empleo, frente a un $17,2 \%$ de los encuestados con contrato por cuenta ajena. Todos los encuestados están de acuerdo en que el factor más destacado a la hora de encontrar empleo es la experiencia con valores que van del 70,7\% para los trabajadores por cuenta propia al $67,1 \%$ para los trabajadores por cuenta ajena.

CUADRo 6.-Distribución porcentual de los tres factores más influyentes a la hora de encontrar empleo

\begin{tabular}{|l|c|c|c|c|c|c|c|}
\hline & Estudios & Experiencia & Contactos & Iniciativa & $\begin{array}{c}\text { Asumir } \\
\text { riesgos }\end{array}$ & Suerte & Otros \\
\hline Cuenta ajena & 60,4 & 67,1 & 57,1 & 55,0 & 17,2 & 45,6 & 34,07 \\
\hline Cuenta propia & 55,7 & 70,7 & 63,7 & 56,7 & 27,9 & 42,1 & 34,11 \\
\hline
\end{tabular}

\section{AsPeCtOS VALORADOS DEL TRABAJO Y SATISFACCIÓN CON EL MISMO}

Los valores del trabajo han sido definidos de forma diversa por distintos autores. Unos lo definen como cualidades que las personas desean de su trabajo, siendo más fundamentales que los intereses (Super, 1970). Otros los consideran como 
cualidades que muestran una relación entre la necesidad activada y su satisfacción (Zytowski, 1970).

Un componente esencial del concepto multidimensional del significado del trabajo es el relativo a los aspectos valorados del trabajo. Para abordar su análisis, el grupo de investigación Meaning of Work (MOW, 1987) toma en consideración que sea un trabajo interesante, el ajuste de habilidades y requerimientos del puesto, la variedad, la autonomía, el sueldo, las oportunidades de aprender, la estabilidad y las relaciones interpersonales.

Para el estudio de la importancia del trabajo para los jóvenes se han utilizado indicadores para evaluar la importancia o centralidad del trabajo. Estos indicadores tratan de determinar la centralidad relativa del trabajo para los encuestados dando valores que oscilan entre uno como nada importante, hasta siete como muy importante.

Los aspectos que se han analizado hacen referencia a fenómenos que acompañan a la actividad laboral, pero que no forman parte inherente de la misma. Concretamente, se han tomado en consideración los aspectos contractuales de la actividad laboral (como la estabilidad en el trabajo), las compensaciones (como el salario, o el tiempo de vacaciones) y las oportunidades de desarrollo de la carrera.

En el cuadro 7 se muestran las valoraciones por tipo de contrato. En primer lugar, es interesante constatar la elevada valoración de todos los aspectos extrínsecos (por encima del 5,77 en una escala de 1 a 7). Pese a que existen ligeras diferencias dentro de cada tipo de contrato, el ranking valorativo es muy similar en cada uno de los ítems analizados. Destaca el hecho de que los aspectos menos valorados por los encuestados sean las vacaciones con un valor de 5,77 para los autónomos, frente a un valor de 5,97 para los trabajadores por cuenta ajena.

CUADRo 7.-Grado de importancia de los siguientes aspectos del trabajo

\begin{tabular}{|l|c|c|}
\hline & Cuenta ajena & Cuenta propia \\
\hline Vacaciones & 5,97 & 5,77 \\
\hline Contactos & 6,00 & 6,22 \\
\hline Promoción & 6,17 & 6,14 \\
\hline Horario & 6,33 & 5,96 \\
\hline Iniciativa & 6,26 & 6,42 \\
\hline Jefe que apoye & 6,35 & 6,23 \\
\hline Estabilidad & 6,55 & 6,47 \\
\hline Salario & 6,55 & 6,51 \\
\hline Oportunidades de aprender & 6,53 & 6,61 \\
\hline Total & 6,30 & 6,26 \\
\hline
\end{tabular}


Se ha calculado el valor promedio de todos los aspectos extrínsecos considerados observando que la importancia es ligeramente superior para los trabajadores con contrato por cuenta ajena $(6,30)$ frente a los trabajadores con contrato por cuenta propia $(6,26)$.

Conocer el nivel de bienestar psicológico general de una sociedad es importante, pero mucho más aún en el caso de los jóvenes pues, tal como sugiere la literatura, las características personales claves en la inserción laboral como son el tener iniciativa o la pasividad, están relacionadas con su nivel de bienestar psíquico, al igual que con su bienestar económico y con el trabajo.

La satisfacción laboral se ha definido como «una actitud global de carácter más o menos positivo ante diferentes aspectos de la experiencia laboral. Esa actitud implica una apreciación de la situación del trabajo en cada aspecto considerado y del grado en que esa situación responde a las expectativas y aspiraciones del joven» (García-Montalvo y Peiró, 2001). En la medida en que la situación real responda a las expectativas y aspiraciones el nivel de satisfacción será elevado, en la medida en que no lo haga, la satisfacción será baja o provocará insatisfacción.

En el cuadro 8 se muestran los resultados obtenidos para cada una de las facetas consideradas: estabilidad en el empleo, el horario, el salario, oportunidades de promoción, vacaciones, contactos personales y las oportunidades de desarrollo de carrera. Es interesante señalar, en primer lugar, que la faceta extrínseca que produce una satisfacción más elevada para los encuestados autónomos es el poder tener iniciativa (con una valoración de 6,19 en una escala de 1 a 7) y la que menos satisfacción les produce son las vacaciones con sólo un 3,93. Mientras que en el caso de los trabajadores por cuenta ajena, la faceta que les produce más satisfacción es la estabilidad y el horario con valores de 5,49 y la que menos las oportunidades de promoción con un valor de 4,88. Destaca el hecho de que los trabajadores por cuenta propia se encuentren más satisfechos, en general, con los aspectos extrínsecos de su trabajo $(5,56)$ que los trabajadores por cuenta ajena $(5,32)$.

CuAdro 8.-Grado de satisfacción con los siguientes aspectos del trabajo

\begin{tabular}{|l|c|c|}
\hline & Cuenta ajena & Cuenta propia \\
\hline Vacaciones & 5,04 & 3,93 \\
\hline Promoción & 4,88 & 5,47 \\
\hline
\end{tabular}


CUADRo 8.-Grado de satisfacción con los siguientes aspectos del trabajo (cont.)

\begin{tabular}{|l|c|c|}
\hline Salario & 5,04 & 5,19 \\
\hline $\begin{array}{l}\text { Oportunidades de } \\
\text { aprender }\end{array}$ & 5,40 & 5,80 \\
\hline Horario & 5,49 & 5,59 \\
\hline Iniciativa & 5,37 & 6,19 \\
\hline Estabilidad & 5,49 & 5,81 \\
\hline Jefe que apoye & 5,46 & 6,15 \\
\hline Contactos & 5,72 & 5,93 \\
\hline Total & 5,32 & 5,56 \\
\hline
\end{tabular}

\section{ESTIMACIONES}

Con el fin de analizar si los procesos de incorporación de los jóvenes al mercado laboral, así como los aspectos valorados del trabajo y su satisfacción con el mismo, son significativos a la hora de impulsar la decisión individual de ser emprendedor, hemos utilizado un modelo logit binario ${ }^{1}$ en donde la variable dependiente es el tipo de relación laboral (valor de referencia: ser autónomo) y las independientes son aquellas que consideramos pudieran ser relevantes teniendo en cuenta el estudio descriptivo (ver anexo).

CUADRo 9.--Modelo logit binomial de elección de ser emprendedor

\begin{tabular}{|l|l|c|c|c|c|}
\hline Variables & \multicolumn{1}{|c|}{ B } & E.T. & Wald & Sig. & Exp(B) \\
\hline Características generales & & & & & \\
\hline Edad & & & & & \\
\hline Entre 16 y 19 años & $-1,690$ & 0,754 & 5,026 & 0,025 & 0,185 \\
\hline Entre 30 y 34 años & 0,926 & 0,291 & 10,138 & 0,001 & 2,524 \\
\hline Estado civil & & & & & \\
\hline Tener pareja & $-1,072$ & 0,374 & 8,195 & 0,004 & 0,342 \\
\hline Actividad & & & & & \\
\hline Construcción & 1,307 & 0,389 & 11,290 & 0,001 & 3,696 \\
\hline
\end{tabular}

${ }^{1}$ El objetivo primordial que resuelve esta técnica es el de modelar cómo influye en la probabilidad de aparición de un suceso, habitualmente dicotómico (como en nuestro caso), la presencia o no de diversos factores y el valor o nivel de los mismos. 
CuAdro 9.--Modelo logit binomial de elección de ser emprendedor (cont.)

\begin{tabular}{|l|l|l|l|l|l|}
\hline Inserción laboral & & & & & \\
\hline Búsqueda de empleo & & & & & \\
\hline Currículum & $-0,845$ & 0,278 & 9,256 & 0,002 & 0,430 \\
\hline Oposiciones & $-2,361$ & 1,027 & 5,281 & 0,022 & 0,094 \\
\hline Autoempleo & 4,124 & 0,456 & 81,659 & 0,000 & 61,779 \\
\hline Valoración del trabajo & & & & & \\
\hline Importancia del trabajo & & & & & \\
\hline Vacaciones & $-0,724$ & 0,281 & 6,625 & 0,010 & 0,485 \\
\hline Satisfacción del trabajo & & & & & \\
\hline \multicolumn{1}{|l|}{ Vacaciones } & $-0,863$ & 0,277 & 9,684 & 0,002 & 0,422 \\
\hline Iniciativa & 1,064 & 0,352 & 9,121 & 0,003 & 2,898 \\
\hline Constante & $-1,703$ & 0,382 & 19,905 & 0,000 & 0,182 \\
\hline $\mathrm{R}^{2}$ de Nagelkerke & & & \multicolumn{2}{|c|}{0,525} & \\
\hline Clasificación correcta del modelo & & & \multicolumn{5}{|c|}{} \\
\hline
\end{tabular}

Para la calibración del modelo se utilizó el test de Hosmer_Lemeshow ${ }^{2}$ en el que se obtuvo una $\chi^{2}$ de 4,839 con 8 grados de libertad y $\mathrm{p}=0,775$, no existieron diferencias estadísticamente significativas entre el tipo de contrato observado y esperado.

Antes de pasar a estudiar los resultados recogidos en el cuadro 9, es importante tener en cuenta las limitaciones de los datos, puesto que de las 2.067 encuestas válidas sólo 1.012 encuestados trabajaban de los cuales 189 tenían un contrato por cuenta propia y 823 por cuenta ajena.

Los resultados de la estimación de nuestro «modelo logit de elección de ser emprendedor», se encuentran recogidos en el cuadro 9 y son los siguientes:

- Se observa una relación negativa entre que el encuestado esté en el rango bajo de edad (de 16 a 19 años) y la probabilidad de que el encuestado sea emprendedor.

${ }^{2}$ La prueba de Hosmer-Lemeshow (1989) consiste en dividir el recorrido de la probabilidad en deciles de riesgo (por ej. la probabilidad de ser emprendedor $\leq 0.1, \leq 0.2, \ldots, \leq 1$ ) y calcular tanto la distribución de emprendedores, como la de no emprendedores prevista por la ecuación y los valores realmente observados. Ambas distribuciones, esperada y observada, se contrastan mediante una prueba de $\chi^{2}$. 
- Por otro lado, existe una relación positiva entre tener más de 30 años y que el encuestado sea emprendedor.

- Con relación al estado civil, el tener pareja afecta de forma negativa a la probabilidad de ser emprendedor.

- En el caso de los jóvenes gallegos de las comarcas estudiadas el hecho de que trabajen en el sector de la construcción afecta positivamente a la probabilidad de que se tenga un contrato por cuenta propia.

- Con relación a la búsqueda de empleo, se detecta que los encuestados que busquen conseguir empleo mediante la preparación de oposiciones tienen una relación negativa (lo que era de suponer) con la probabilidad de trabajar por cuenta propia, lo mismo sucede con los jóvenes que busquen empleo mediante el envío de cartas o currícula a empresas, y, por el contrario, los encuestados cuyo método de búsqueda principal sea el establecerse por su cuenta, tienen más probabilidad de llegar a conseguirlo.

- En relación con la importancia de las características propias del trabajo aquellos encuestados que le den poca importancia a las vacaciones (menor que la media de la muestra) tienen más probabilidad de establecerse por su cuenta.

- Aquellos encuestados cuya satisfacción con la probabilidad de tener iniciativa en el trabajo sea superior a la media afecta positivamente a la probabilidad de ser trabajador por cuenta propia, mientras que los que tengan una satisfacción con las vacaciones superior a la media tendrán una probabilidad menor de ser trabajador por cuenta propia.

\section{CONCLUSIONES}

En este trabajo se realiza una primera aproximación a la relación existente entre los procesos de incorporación al mercado laboral, las estrategias que utilizan los jóvenes en la búsqueda de empleo, así como la importancia y la satisfacción concedidas al trabajo, con el hecho de tomar la decisión de ser emprendedor para los jóvenes gallegos de diversas zonas (Comarcas de Bergantiños y Eume y zonas geográficas de Costa da Morte y Ferrolterra), Los resultados revelan información importante respecto a los factores que afectan a la decisión de ser emprendedor entre los que cabe destacar:

En primer lugar, la mayoría de los jóvenes trabajan por cuenta ajena. Los datos muestran que el $81,32 \%$ de los jóvenes que trabajan tienen un empleo por cuenta ajena, frente al 18,68\% que son autónomos. Las mujeres muestran una preferencia todavía más acentuada que los hombres $(85,84 \%$ frente al $75,17 \%)$. 
En segundo lugar, el colectivo de trabajadores emprendedores muestra diferencias significativas según la edad. En los datos se aprecia una presencia muy alta de los jóvenes de más edad. El 50,3\% tiene entre 30 y 34 años y el 32,4\% está en el tramo de edad de 25 a 29 años.

En tercer lugar, uno de los aspectos básicos de la relación entre el capital humano y los emprendedores lo constituye la dotación de capital humano individual de los miembros de este colectivo. Por niveles educativos es muy significativo que en los emprendedores el nivel educativo más representativo es el de estudios bajos con el $26,88 \%$, mientras que en los trabajadores por cuenta propia son los estudios superiores con el $31,55 \%$.

En cuarto lugar, las estimaciones realizadas del modelo logit en relación a las características generales nos muestran que los jóvenes que se encuentran en el grupo de edad más bajo (de 16 a 19 años) tienen menos probabilidad de ser emprendedor, mientras que aquellos jóvenes con edades comprendidas entre los 30 y 34 años tienen más probabilidades de conseguirlo. Si nos referimos al estado civil, el hecho de tener pareja afecta negativamente a la probabilidad de ser emprendedor. Por el contrario, el hecho de que los jóvenes trabajen en el sector de la construcción afecta positivamente a dicha probabilidad.

En quinto lugar, las estimaciones realizadas en relación a las variables de inserción laboral sugieren que el tipo de búsqueda de empleo influye significativamente en la probabilidad de ser emprendedor. En particular, la búsqueda de empleo mediante el envío de cartas o currícula a empresas y el preparar oposiciones afecta negativamente a la probabilidad de ser emprendedor, mientras que el autoempleo como método de búsqueda de empleo afecta positivamente al hecho de conseguirlo.

Por último, las estimaciones realizadas del modelo logit en relación a las variables de valoración del trabajo, sugieren que la importancia de factores como las vacaciones y la satisfacción que se tenga con las mismas influyen negativamente en la probabilidad de ser emprendedor. Por el contrario, la satisfacción que se tenga con la posibilidad de tener iniciativa influye positivamente en la probabilidad de que el joven se establezca por su cuenta.

\section{REFERENCIAS BIBLIOGRÁFICAS}

Acs, Z. J.; Arenius, P.; Hay, M. y Minniti, M. (2005), «Global Entrepreneurship Monitor 2004, executive report» en http://www. gem-consortium.org/. 
Aldrich, H. y BAKER, T. (1997), «Blinded by the cities? Has there been progress in entrepreneurship research?», en Sexton \& Smilor (eds.), Entrepreneurship 2000, Chicago, Upstart Publishing Company.

Alexei, T. y Kolvereid, L. (1999), «Self-employment intentions among Russian students», Entrepreneurship and Regional Development, 11 (3), 269-271.

Álvarez, P.; Reyna, R. y García del Junco, J. (2004), «Measuring entrepreneurs characteristics», 12th International objetive Measurement, Work-Shop, Kairns, Australia, 2004.

AMit, R.; Glosten, L. y Muller, E. (1993), «Challenges to theory development in entrepreneurship research», Journal of Management Studies, vol. 30, 815-834.

Audet, J. (2002), «A longitudinal Study of the Entrepreneurial Intentions of University Students», Frontiers of Entrepreneurship Research, Babson College, Wellesley.

AudRETSCH, D. B. (2002), Entrepeneurship a survey of the literature, Institute of development strategies, Indiana University and Centre of Political Policy Research (CEPR), Londres.

Baena, J.; Ganaza, J. y García del Junco, J. (2001), «Necesidad de potenciar como recursos estratégico los valores personales en la organización», en La empresa deslocalizada, Las Palmas de Gran Canaria, 478-493.

BARON, R. A. (2002), «OB and Entrepreneurship: the reciprocal benefits of closer conceptual links» en B. M. Staw y R. M. Kramer (eds.), Research in Organizational Behavior-An annual series of analytical essays and critical reviews, 24, Oxford, Elsevier Science, 225-270.

Baumol, W. (1990), «Entrepreneursip: Productive, unproductive, and destructive», Journal of Political Economy, vol. 98, págs. 893-921.

Clark, J. B. (1924), Essential of economic theory, as applied to modern problems of industry and public policy, Nueva York, McMillan Co.

Comisión de las Comunidades Europeas (2003), Informe de síntesis. El debate público suscitado por el Libro Verde sobre el espíritu empresarial en Europa, Bruselas, Comisión Europea.

Congregado y otros (2008), El capital humano y los emprendedores en España, Edt. Instituto Valenciano de Investigaciones Economicas (IVIE).

Cooper, C. y Gimeno-Gascón, F. (1992), «Entrepreneurs, processes of founding and new-firm performance», en Sexton y Kasarda (eds.), The State of Entrepreneurship, Boston, MA, PSW-KENT, Publishing Co., 301-340.

CuAdrado, J. R. (coord.) (2004), Empleo autónomo y empleo asalariado, Subdirección General de Publicaciones del Ministerio de Trabajo y Asuntos Sociales, Madrid.

Davidsson, P. y Honig, B. (2003), «The role of social and human capital among nascent entrepreneurs», Journal of Business Venturing, vol 18, 301-331.

DíAz, C. (2002), La creación de empresas. Revisión de teorías y escuelas, Ediciones La Coria, Fundación Xavier de Alas, Trujillo. 
Douglas, E. J. y ShePherd, D.A. (2002), «Self-Employment as a Career Choice: Attitudes, Entrepreneurial intentions, and utility Maximization», Entrepreneurship Theory and Practice, 26 (3), 81-90.

Evans, D. y Jovanovic, B. (1989), «An estimated model of entrepreneurial choice under liquidity constraints», J. Polot. Econom, vol 97, 4, 808-827.

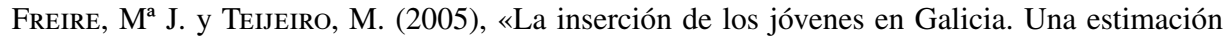
Minceriana de la rentabilidad de la educación», Cuadernos de Economía, vol. 28, n. ${ }^{\circ}$ 77-78, 57-80.

- (2008), «Valores laborales y actitudes de los jóvenes de las comarcas gallegas hacia el empleo», en Investigaciones de Economía de la Educación, 3, 375-382.

García, A. y Wandosell, G. (2004), «Motivaciones y obstáculos en la creación de empresas. Efectos de la experiencia empresarial», Boletín Económico del ICE, n. ${ }^{\circ} 2819,19-36$.

García del Junco, J.; Martín, F. y Álvarez, P. (2006), «Los valores personales y su influencia en el espíritu del emprendedor», Boletín de Estudios Económicos, vol. LXI (189), 471-494.

García del Junco, J.; Reyna, R. y Álvarez, P. (2003), «Can the entrepreneurship be measured?», Working Paper Strategy Research Conference, Harvard Business School, Harvard University, Boston.

García Montalvo, J. (2001), Formación y empleo de los graduados superiores en España y Europa, Fundación Bancaja-Ivie, Valencia.

GARTNER, W. B. (1988), «Who is an entrepreneur? Is the wrong question», American Journal of Small Business, 12, 4, 11-32.

- (2002), «Are entrepreneurs different than others?», Arsrapport 2001/2002. Syddansk Universitets trykkeri, 11-15.

Heshmati, A. (2001), «On the growth of Micro and Small Firms: Evidence from Sweden», Small Business Economics, vol. 17, n. ${ }^{\circ} 3,213-228$.

Hoнті, S. (2000), «Job flows and job quality by establishment size in the finish manufacturing sector, 1980-1994», Small Business Economics, vol. 15, n. ${ }^{\circ}$ 4, 265-281.

IbrAYeBA, E. (1999), «Entrepreneurship in transitionary economies: testing a social cognitive model», Tesis doctoral, University of Nebraska, Lincoln.

Johnson, S. y Loveman, G. (1995), Starting over in Eastern Europe: Entrepreneurship and economic renewal, Boston, Harvard Business School Press.

Khilstrom, R. y LAFFont, J. (1979), «A general equilibrium entrepreneurial theory of firm formation based on risk aversion», J. Polit. Econom, vol. 87, 4, 719-748

Kirzner (1973), Competition and Entrepreneurship, University of Chicago Press, Chicago, IL.

Krueger, N. F., Jr. y Dickson, P. R. (1994), «How believing in ourselves increases risk taking: Perceived self-efficacy and opportunity recognition», Decision Sciences, 25, 385-400. 
Low, M. y Macmillan, I. (1988), «Entrepreneurship: Past research and future challenges», Journal of Management, 14, 2, 139-161.

Macmillan, I. y Katz, J. (1992), «Idiosyncratic milieus of entrepreneurial research: The need for comprehensive theories», Journal of Business Venturing, 7, 1, 1-8.

Marshall, A. (1980), Principles of economics, MacMillan, Guadarrama.

Mises, L. V. (1949), La acción humana un tratado de economía, Madrid, Unión Editorial.

Morales, C. E. y Rahe, M. (2007), «Las competencias para el éxito de los emprendedores y de las emprendedoras», Harvard Deusto Business Review, n. ${ }^{\circ}$ 157, págs. 64-71.

Moriano, J. A.; Palací, F. J. y Morales, J. F. (2006), «El perfil psicosocial del emprendedor universitario», Revista de Psicología del Trabajo y de las Organizaciones, vol. 22, 1, 75-99.

MOW Internacional Research Team (1987), The meaning of working, Londres, Academia Press.

NAFFZINGER, M. (1998), «Entrepreneurship: a person based theory approach», en Katz y Brockhaus (eds.), Advances in entrepreneurship firm emergence and growth (II), Greenwich Ja Press, 21-50.

PAIGE, R. (1999), Craft retail entrepreneurs perceptions of success and factor affecting success, Iowa States University.

Peiró, J. M. y Moret, D. (eds.) (1987), Socialización laboral y desempleo juvenil. La transición de la escuela al trabajo, Nau Llibres, Valencia.

Roure, C. L.; González, N. y Nieto, M. (2007), «Oportunidades innovadoras y tecnológicas de la creación de empresas», Ed. Universidad de La Rioja, en Conocimiento, Innovación y Emprendedores: Camino al futuro.

SÁnchez-Almagro, M. L. (2003), «Perfil Psicológico del Autoempleado», Tesis doctoral, Universidad Complutense de Madrid.

SCHumpeter, J. A. (1936), The theory of economic development: an inquiry into profits, capital credit, interest, and business cycle, Cambridge, Harvard University Press.

Shane, S. A. y Venkataraman, S. (2000), «The promise of entrepreneurship as a field of research», Academy of Management Review , vol. 25, 217-226.

Shaver, K. G. y Scott, L. R. (1991), «Person, process, and choice: the psychology of new venture creation», Entrepreneurship Theory and Practice, winter, 23-42.

Slevin, D. y CoIn, J. (1995), «Entrepreneurship as firm behaviour. A research model», en Katz y Brochaus (eds.), Advances in entrepreneurship firm emergence and growth, Greenwich. Connecticut.

SuPER, D. E. (1970), Work Values Inventory, Boston, Houghton Mifflin.

Toledano, N. (2003), Crear empresas, Diputación de Huelva, Servicio de Publicaciones y área de Desarrollo Local, Huelva. 
VeCiana, J. M. (1999), «Creación de empresas como programa de investigación científica», Revista Europea de Dirección y Economía de la Empresa, vol. 8, 3, 11-36.

ZAHARA, S. (1991), «Predictors and financial outcomes of corporate entrepreneurship: an exploratory study», Journal of Business Venturing, 6, 4, 259-285.

Vennekers, A. R. y Thurik, A. R. (1999), «Linking Entrepeneurship and Economic Growth», Small Business Economics, 13, págs. 27-55.

Zytoeski, D. G. (1970), Pshicological Influences on Vocational Development, Nueva York, Houghton Miffin.

\section{APÉNDICE}

\section{Definición de variables}

A continuación explicamos cómo se han construido las variables que hemos utilizado en el modelo de elección discreta.

Variables de características generales:

- sexo: la categoría de referencia es ser hombre.

_ edad_1: Variable dicotómica cuyo valor de referencia es que la edad del individuo sea menor o igual a 19 años.

— edad_2: Variable dicotómica cuyo valor de referencia es que la edad del individuo esté entre 20 y 24 años.

—_ edad_3: Variable dicotómica cuyo valor de referencia es que la edad del individuo esté entre 25 y 29 años.

- edad_4: Variable dicotómica cuyo valor de referencia es que la edad del individuo esté entre 30 y 34 años.

—_civil_1: El estado civil siendo la categoría de referencia tener pareja.

— est_3.1: Variable dicotómica cuyo valor de referencia es poseer un nivel de estudios bajo.

— est_3.2: Variable dicotómica cuyo valor de referencia es poseer un nivel de estudios medio.

— est_3.3: Variable dicotómica cuyo valor de referencia es poseer un nivel de estudios anterior al superior.

— est_3.4: Variable dicotómica cuyo valor de referencia es poseer un nivel de estudios superior. 
- actividad_1: Variable dicotómica cuyo valor de referencia es que el individuo trabaje en el sector de la agricultura.

— actividad_2: Variable dicotómica cuyo valor de referencia es que el individuo trabaje en el sector de la construcción.

_ actividad_3: Variable dicotómica cuyo valor de referencia es que el individuo trabaje en el sector del comercio y restaurante.

— actividad_4: Variable dicotómica cuyo valor de referencia es que el individuo trabaje en el sector de las finanzas.

- exper_5: Variable dicotómica cuyo valor de referencia es que el individuo posea una experiencia laboral menor o igual a 5 años.

- exper_10: Variable dicotómica cuyo valor de referencia es poseer una experiencia laboral de 5 a 10 años.

- exper_15: Variable dicotómica cuyo valor de referencia es poseer una experiencia laboral de más de 10 años.

Variables de inserción laboral:

- a10_1: Variable dicotómica cuyo valor de referencia es que la forma de búsqueda de empleo sea el INEM.

— a10_2: Variable dicotómica cuyo valor de referencia es que la forma de búsqueda de empleo sea el currículum.

— a10_3: Variable dicotómica cuyo valor de referencia es que la forma de búsqueda de empleo sea preparar oposiciones.

- a10_4: Variable dicotómica cuyo valor de referencia es que la forma de búsqueda de empleo sea promover los contactos.

- a10_5: Variable dicotómica cuyo valor de referencia es que la forma de búsqueda de empleo sea intentar establecerse por su cuenta.

- a10_6: Variable dicotómica cuyo valor de referencia es que la forma de búsqueda de empleo sea por otros medios.

— b5_1: Variable dicotómica cuyo valor de referencia es que los estudios influyen a la hora de encontrar un empleo.

— b5_2: Variable dicotómica cuyo valor de referencia es que la experiencia influye a la hora de encontrar un empleo.

— b5_3: Variable dicotómica cuyo valor de referencia es que los contactos influyen a la hora de encontrar un empleo.

— b5_4: Variable dicotómica cuyo valor de referencia es que el poseer iniciativa influye a la hora de encontrar un empleo. 
— b5_5: Variable dicotómica cuyo valor de referencia es que el hecho de asumir riesgos influye a la hora de encontrar un empleo.

- b5_6: Variable dicotómica cuyo valor de referencia es que el factor suerte influye a la hora de encontrar un empleo.

Variables relacionadas con la valoración del trabajo y la satisfacción del mismo:

- i_horario: Variable dicotómica cuyo valor de referencia es que la importancia del horario del trabajo sea superior a la media del total de factores extrínsecos considerados.

- i_vacaciones: Variable dicotómica cuyo valor de referencia es que la importancia de las vacaciones sea superior a la media del total de factores extrínsecos considerados.

- i_contactos: Variable dicotómica cuyo valor de referencia es que la importancia de los contactos en el trabajo sea superior a la media del total de factores extrínsecos considerados.

- i_iniciativa: Variable dicotómica cuyo valor de referencia es que la importancia de poder tener iniciativa en el trabajo sea superior a la media del total de factores extrínsecos considerados

- s_horario: Variable dicotómica cuyo valor de referencia es que la satisfacción con el horario del trabajo sea superior a la media del total de ítemes analizados.

- s_vacaciones: Variable dicotómica cuyo valor de referencia es que la satisfacción con las vacaciones sea superior a la media del total de ítemes analizados.

- s_contactos: Variable dicotómica cuyo valor de referencia es que la satisfacción con los contactos en el trabajo sea superior a la media del total de ítemes analizados.

- s_iniciativa: Variable dicotómica cuyo valor de referencia es que la satisfacción con la posibilidad de tener iniciativa en el trabajo sea superior a la media del total de ítemes analizados. 